\title{
Liothyronine use in primary hypothyroidism - current concepts
}

\author{
Marcio J. Concepción-Zavaleta ${ }^{\circledR 1}$, Sofía P. Ildefonso-Najarro ${ }^{\circledR 2}$, José L. Paz-Ibarra ${ }^{\circledR 3}$, \\ Freddy V. Fernández-Dávila ${ }^{\circledR 2}$, Diana C. Deutz-Gómez², Katia E. Rivera-Fabián², \\ Ramiro G. Herrera-Cabezas ${ }^{4}$, Luis A. Concepción-Urteaga $\mathbb{1}^{5}$
}

${ }^{1}$ Division of Endocrinology, Clínica Stella Maris, Lima, Perú

${ }^{2}$ Division of Endocrinology, Hospital Nacional Guillermo Almenara Irigoyen, Lima, Perú

${ }^{3}$ Division of Endocrinology, Hospital Nacional Edgardo Rebagliati Martins, Lima, Perú

${ }^{4}$ Division of Internal Medicine, Hospital Nacional Guillermo Almenara Irigoyen, Lima, Perú

${ }^{5}$ School of Medicine, Universidad Nacional de Trujillo, Perú

\begin{abstract}
Hypothyroidism is an endocrine disorder whose management raises many challenges in clinical practice. Its standard treatment is levothyroxine (LT4). The goal of the treatment is to normalize signs and symptoms, as well as to achieve thyroid-stimulating hormone (TSH) concentrations within the reference range, on an individual basis. It is known that $5-10 \%$ of hypothyroid patients remain symptomatic, despite achieving the target TSH levels, which, in turn, affects their quality of life. After ruling out other causes of non-thyroid origin for this persistence, it is suggested that these patients could benefit from the use of liothyronine (LT3), added to LT4, especially if polymorphism of the deiodinase 2 (D2) genes is documented. There exist a variety of LT3 preparations, whose concentrations vary from 5 to 50 ug, with the recommended LT4/LT3 ratio of 13:1-20:1. The goals of combination therapy should be to achieve a physiological ratio of free triiodothyronine/free thyroxine (FT3/FT4) and non-suppression of TSH. Because there is currently no guide that makes evidence-based recommendations on the use of LT3 in primary hypothyroidism, more clinical studies are needed to be able to identify hypothyroid patients who may benefit from the use of LT3, by identifying new biomarkers. (Endokrynol Pol 2021; 72 (6): 652-660)
\end{abstract}

Key words: liothyronine; levothyroxine; hypothyroidism; deiodinase

\section{Introduction}

Hypothyroidism, especially primary hypothyroidism, has been identified as one of the most common endocrine pathologies, affecting $3 \%$ of the general population, of which $5.1 \%$ are women and $0.9 \%$ men [1]. In Latin America, the estimated prevalence is 10\% [2], and there are no current studies on the epidemiology of this disease in our country.

Before the 1970s, both liothyronine (LT3) and levothyroxine (LT4) combined with thyroid hormone replacement therapy and desiccated thyroid extract (DTE) were widely prescribed. However, the discovery that thyroxine (T4) is largely converted to triiodothyronine (T3) outside the thyroid, within target tissues, and concerns about dosage and stability of free T3 (FT3) profiles in patients treated with LT3 or DTE have made LT4 the standard therapy for almost 50 years [3]. No expert guidance or recommendation has suggested thyroid hormone replacement with LT3 alone, but the debate has been whether combined therapy with LT4 and LT3 might be better than LT4 alone.
It has been found that $5-10 \%$ of hypothyroid patients receiving LT4 continue to experience disabling symptoms such as fatigue, depression, and impaired cognition, despite being adequately replaced biochemically, affecting their quality of life [4]. In a study that aimed to evaluate satisfaction and quality of life (QOL) in 969 hypothyroid patients through a survey, it was found that $77.6 \%$ were dissatisfied, and the scores of QOL were low. Furthermore, combined treatment of LT4 + LT3 or with DTE was found to be associated with a better QOL compared to LT4 monotherapy. However, multivariate analysis failed to confirm an association between the type of thyroid hormone treatment and QOL or satisfaction [5]. In addition, Peterson et al. performed an online survey for 12,146 hypothyroid patients and found, strikingly, that individuals taking DTE reported a higher median treatment satisfaction of 7 out of 10 , the LT4 treatment group exhibited satisfaction of 5 , and for the LT4 + LT3 treatment group the satisfaction level was 6 . These results suggest that LT4+LT3 treatment is still not satisfactory, but more studies are needed to confirm this finding [6]. 
However, due to the controversy over whether hypothyroid patients can be optimally treated with LT4 alone, numerous studies over time have addressed the potential benefits of combination therapy, but these have been inconclusive. Recent genetic studies have shown the importance of deiodinases in maintaining the euthyroid state, as well as the growing evidence of polymorphisms of these, which would affect the conversion of T4 to T3 [7], providing a solid argument for the addition of LT3 to the therapy.

Regarding the benefits of LT3, a study compared LT3 therapy with LT4 therapy and demonstrated the benefits of LT3 therapy, i.e. weight loss and favourable changes in the lipid profile, which could be relevant to hypothyroid patients affected by comorbid conditions such as cardiovascular diseases, diabetes, dyslipidaemia, and obesity, where weight control and lowering of serum cholesterol are particularly important [8].

However, currently, the prolonged use of LT3 can be a challenge because it needs to be administered multiple times a day, which can affect adherence [8]; in addition, LT3 is more expensive compared to LT4, which has caused a generalized variability in patient access to it [9]. Therefore, it is important to select patients who would potentially benefit from combined LT3 and LT4 therapy.

It is of interest that systematic and comprehensive research have demonstrated the potential benefits of LT3 therapy, because endocrinologists are frequently consulted about hypothyroid adult patients taking LT4 who are dissatisfied with their therapy. Therefore, once non-thyroid causes related to these symptoms have been excluded, the patient and their doctor may consider some therapies, among them the use of combined therapy with LT4 and LT3, whose use remains controversial because it bears contradictory results in most published clinical trials [10].

Thus, in this article, we aimed to carry out an updated narrative review of the literature on the use of
LT3 in the treatment of primary hypothyroidism, in order to establish which patients may be eligible for combined LT3 and LT4 therapy.

\section{Pathophysiology}

Thyrotropin-releasing hormone (TRH)-synthesizing neurons are present in several nuclei of the hypothalamus, but only hypophysiotrophic TRH neurons, located in the paraventricular nucleus, have been determined to be involved in the regulation of the hypothalamic-pituitary-thyroid (HPT) axis [11]. TRH stimulates the synthesis and release of TSH in the thyrotrophic cells of the adenohypophysis, where it is transported through the pituitary portal system. TSH, in turn, stimulates thyroid follicular cells to release $\mathrm{T} 4$ $(80 \%)$ and $\mathrm{T} 3(20 \%)$ [12].

The negative feedback of thyroid hormones occurs in the pituitary and in the hypothalamus; consequently, the production and secretion of TSH and TRH are regulated by the levels of circulating thyroid hormones. In this way, the HPT axis regulates the concentration of thyroid hormones [11, 13].

Thyroid hormones are then transported into the cells in order to exert their effects. Once transported, only $\mathrm{T} 3$ binds to the thyroid hormone receptor in the nucleus, while the pro-hormone T4 needs to be converted into active hormone T3 by deiodinases [11]. In euthyroid individuals, the peripheral conversion of $\mathrm{T} 4$ to T3 accounts for $80 \%$ of the T3 produced [12].

The deiodination of thyroid hormone is often catalysed by the family of selenoenzymes called deiodinases (D) (Tab. 1), which comprises three enzymes: type 1 (D1), type 2 (D2), and type 3 (D3) [11]. Both the inner ring (phenolic) and the outer ring (tyrosyl) of T4 can be deiodinated, which ultimately leads to the formation of 3,3'-diiodotyrosine. The key to this process is the action of deiodinases that are subspecialized in differ-

Table 1. Main characteristics of deiodinases type 1, 2, and 3

\begin{tabular}{lccc}
\hline & D1 & D2 & D3 \\
\hline Preferred substrate & T3r & T4 & T3 \\
\hline Tissue localization & Liver, kidney, and thyroid gland & $\begin{array}{c}\text { Pituitary, CNS, brown adipose } \\
\text { tissue, skin, and heart }\end{array}$ & CNS, skin, and placenta \\
\hline Cellular localization & Plasma membrane & Endoplasmic reticulum & Plasma membrane \\
\hline Hyperthyroidism & $\uparrow$ & $\downarrow$ & $\uparrow$ \\
\hline Hypothyroidism & $\downarrow$ & $\uparrow$ & $\downarrow$ \\
\hline Selective activator & None & cAMP & Hypoxia \\
\hline Selective inhibitor & Propylthiouracil & None & None \\
\hline Clinical relevance & $\begin{array}{l}\text { Responsible of 20\% of } \\
\text { extrathyroidal T3 production }\end{array}$ & $\begin{array}{c}\text { Responsible of 80\% of } \\
\text { extrathyroidal T3 production }\end{array}$ & $\begin{array}{c}\text { Contributes to the main clearance } \\
\text { of T3 }\end{array}$ \\
\hline
\end{tabular}

CNS — central nervous system; cAMP — cyclic adenosine monophosphate; rT3 — reverse T3 
Table 2. Practical method to calculate the appropriate dose of levothyroxine (LT4) and liothyronine (LT3) in eligible patients for combination therapy

\begin{tabular}{|c|c|c|c|}
\hline LT4 dose that normalizes TSH $=X$ & $X=50 \mu \mathrm{g}$ & $X=100 \mu \mathrm{g}$ & $X=150 \mu \mathrm{g}$ \\
\hline $\begin{array}{l}\text { LT3 dose }=Y \\
\text { Formula: } Y=X / 20\end{array}$ & $2.5 \mu \mathrm{g}$ & $5 \mu \mathrm{g}$ & $7.5 \mu \mathrm{g}$ \\
\hline $\begin{array}{l}\text { LT4 dose }=Z \\
\text { Formula: } Z=X-3 Y\end{array}$ & $42.5 \mu \mathrm{g}$ & $85 \mu \mathrm{g}$ & $127.5 \mu \mathrm{g}$ \\
\hline Rounded LT4 dose & $50 \mu \mathrm{g}$ & $88 \mu \mathrm{g}$ & $125 \mu \mathrm{g}$ \\
\hline Ratio LT4/LT3 & 17:1 & 17:1 & 17:1 \\
\hline
\end{tabular}

ent tissues. Most of the T3 in the circulation is derived from the actions of D1 in the liver, kidneys, and thyroid [11]. However, in the hypothalamus and pituitary thyrotroph, D2 allows the feedback loop to recognize T4 concentrations because $\mathrm{T} 4$ is rapidly converted to T3 in these cells [14]. In the nuclei of the anterior pituitary, approximately half of the T3 bound to nuclear receptors is derived from circulating T3, while the remaining half is derived from the intrahypophyseal conversion of T4 to T3 [11].

The major steps in the actions of $\mathrm{T} 4$ are initiated by the entry of T4 into the cell, which is now recognized to be controlled by the expression of various membrane transporters for thyroid hormones [11, 13, 15]. Then, it must be converted to T3 in cells that express D1 or D2, allowing a dual source of T3 (systemic and local) to the HPT axis. After transport into the cell, cytosolic T3 then enters cell nuclei on the HPT axis by pathways yet to be defined; then, it binds to high-affinity chromatin-bound proteins, predominantly the thyroid hormone receptor $\beta 2$ [10]. Due to the rapid generation of T3 from T4 within these cells by D2, the cytosolic T3 concentration is higher, and it remains in a static imbalance with serum T3 [11].

D3 inactivates T3 and is vital during growth and development and later in adult life, because D3 is involved in the development of the placenta, uterus, and embryo/foetus, protecting it from maternal T3 and T4. This allows the autonomous developmental program in the embryo and foetus to control the differentiation process $[11,13,15]$.

Several single-nucleotide polymorphisms have been identified in the genes for deiodinases, and relationships between hormone levels have been investigated. Theoretically, polymorphisms could result in lower enzyme activity and therefore lower conversion of T4 to T3 in the periphery, with even small changes in hormone levels that have important long-term consequences [16].

The D1 (Cys785Thr) polymorphism is associated with higher T4 and reverse T3 (T3r) and lower T3 in plasma, suggesting decreased D1 activity. Conversely, the D1 (Ala1814Gly) polymorphism has been associated with increased plasma T3 levels and a higher T3:T3r ratio, which suggests greater D1 activity. In particular, the D3 (Thr154Gly) polymorphism does not affect the plasma levels of thyroid hormones [17].

The D2 polymorphism (Thr92Ala) is associated with a high T4, a lower T3 in plasma, and a lower T3:T4 ratio, suggesting decreased D2 activity. It is the most investigated because of its clinical relevance. There are several reports that describe its association with clinical syndromes that include hypertension, type 2 diabetes, mental disorders, lung injury, bone turnover, and autoimmune thyroid disease [18]. Studies in pituitary cell and skeletal muscle cell cultures demonstrated that the Thr92Ala enzyme is less efficient in converting T4 to T3. In a study conducted using knock-in mouse models, Thr92Ala-substituted mice manifested hypothyroidism in different areas of the brain, which reversed with LT3 administration [17]. Additionally, the presence of the D2 polymorphism (ORFa-Gly3Asp) has been found to be associated with reduced levels of T4 and reverse T3 (rT3); consequently, they generate an increase in the T3:T4 and T3:rT3 ratio, conditions that reflect an increase in D2 activity [17].

Individuals with such polymorphisms would potentially require LT3 replacement to achieve euthyroidism. However, there remains no clear evidence supporting the relationships between D2 gene polymorphisms, thyroid parameters, and negative clinical consequences [16].

\section{Comparison of liothyronine vs. levothyroxine treatment}

There is a paucity of data to support the use of LT3. However, in some specific situations, they can provide some benefits. In hypothyroid patients with type 2 deiodinase polymorphism, or who are athyreotic ${ }^{19}$, they remain symptomatic despite reaching the target range of the TSH level. Also, in patients with differentiated thyroid cancer, they can be used temporarily for 3-4 weeks, before treatment with radioactive iodine, thus shortening the duration of hypothyroidism [20]. 
LT3 monotherapy has been shown to have metabolic benefits with improvement in weight, LDL cholesterol, mood well-being, and psychometric functioning, as well as being better tolerated in high-risk groups with dyslipidaemia and obesity $[15,21]$.

According to the results of previous studies, patients receiving LT4 replacement therapy have a normal serum TSH, achieve higher total T4 and FT4 concentrations, and lower total T3 and FT3 serum concentrations, compared to healthy controls [22]. FT3 values below the lower limit of normal are observed in approximately $15 \%$ of hypothyroid patients in LT4 [23]. Therefore, it is questionable whether low serum T3 levels may be involved in persistent patient discomfort, requiring higher doses of LT4 to normalize T3 levels.

The mechanism responsible for low serum T3 during LT4 therapy is related to D2. This enzyme catalyses the conversion of T4 to T3 in extra thyroid tissues, thus maintaining normal serum T3 levels during LT4 replacement therapy. D2 has a short half-life (60 minutes), which becomes even shorter (20 minutes) upon interacting with $\mathrm{T} 4$, resulting in ubiquitination of D2 and stimulation for its proteasomal degradation [24]. Therefore, while, in the rest of the body, the production of T3 mediated by D2 progressively decreases with increasing doses of LT4, due to the ubiquitination of D2, this does not occur in the hypothalamus/pituitary, where the dose of LT4 required to normalize TSH is less than the dose that normalizes serum T3. It follows that to achieve a normal serum $\mathrm{T} 3$ concentration, a relatively high dose of LT4 must be administered, which is likely to suppress serum TSH [25], which then provides a justification for LT3 supplementation.

Studies in rats required a combination of LT4 and LT3 replacement to achieve physiological tissue serum concentrations. However, similar studies in humans did not appear to result in an improvement in patient-reported outcomes. In another study, serum T4 and T3 concentrations were not different between responding and non-responding patients to LT3 therapy. This may be because intracellular T3 levels are dependent on several factors, including membrane transport proteins, deiodination, nuclear protein binding (retinoid $\mathrm{X}$ receptor), and thyroid hormone receptors, which are downregulated by serum levels of thyroid hormones [26].

Meta-analyses have compared the use of LT3 alone or in combination with LT4, against LT4 alone, demonstrating that there is no consistent benefit on the use of LT3 over LT4 alone and that there is no difference in terms of adverse effects. Current forms of oral LT3 cause transient unphysiological elevated serum T3 concentrations after dosing, which are not believed to cause low serum TSH concentrations but still may be harmful. More flexible dosing regimens of LT3, with the help of the introduction of 5-ug tablets, make its dosing easier and potentially safer, if they reduce the risk of increased serum T3 [26].

LT3 monotherapy appears more favourable for patients affected by dyslipidaemia and obesity, but there is insufficient evidence to suggest its superiority to standard LT4 therapy, particularly due to the need for strict adherence to the regimen and timing of drug administration, the risk of overdosing or underdosing, and the risk of cardiac and skeletal toxicity. Therefore, the careful and judicious use of LT3 may be indicated in some patients who continue to report adverse symptoms that cannot be attributed to another cause [27].

\section{Comparison of combined levothyroxine + liothyronine therapy vs. levothyroxine monotherapy}

Generally, the rationale for LT4 treatment is that both D1 and D2 convert T4 to T3, thus restoring the T3 pool and clinical euthyroidism [28].

However, as previously explained, LT4 therapy in hypothyroid rats did not achieve normal serum T3 levels, nor did it result in normal T3 levels in all tissues sampled, but it did show restoration of normal tissue concentrations of thyroid hormones with combined therapy with LT4 and LT3 [21, 28].

Therefore, in murine models, TSH secretion has been noted to normalize before T3 levels are fully restored in plasma and other tissues, resulting in low circulating T3 levels and "tissue hypothyroidism". The use of combined therapy with LT4 and LT3 in hypothyroid rats has also normalized serum TSH; moreover, it also corrects the T3 deficiency in peripheral tissues. Consistent with this, markers of euthyroidism, such as serum cholesterol levels, mitochondrial content, and enzymatic activity in liver and skeletal muscle, were closer to values in control rats when administration of LT4 and LT3 was sustained [25].

These conclusions reveal doubts about human replacement therapy with LT4 alone, because it may not be able to restore euthyroidism in all tissues of hypothyroid patients [28].

It is well established that there are two changes in thyroid hormone levels that occur in hypothyroid patients receiving LT4 monotherapy. These changes are as follows: increased FT4 levels and increased FT4/T3 ratio (often associated with a decrease in T3 or FT3 levels). Depending on the study, mean serum T3 or FT3 levels may be in the lower half of the normal range, and levels may even be below the normal range in up to $15 \%$ of individuals [15, 29, 30, 31].

All these differences have been noted to occur despite comparable serum TSH levels. In fact, in 
a cross-sectional study based on the data from the US National Health and Nutrition Examination Survey, the serum levels of T3 and FT3 were 10\% lower in LT4-treated patients than in a control group matched for sex, age, ethnicity, and TSH levels [32]. Therefore, although LT4 therapy is significantly effective in normalizing serum $\mathrm{TSH}$ levels, it fails to restore euthyroidism in some tissues, based on several metabolic parameters such as serum cholesterol levels and other biomarkers that remain abnormal in patients treated with LT4, despite normal serum TSH [32-34].

To date, 13 randomized clinical trials (RCTs) have been published comparing the efficacy of combined LT4 and LT3 therapy with LT4 monotherapy [9]. Four meta-analyses of these combination therapy trials found no clear benefit over LT4 monotherapy in terms of mood, health-related quality of life, or cognitive function. These data have been taken to suggest that at the population level, there is no benefit of using combination therapy over monotherapy. However, none of the studies have achieved physiological replacement [3].

It is noteworthy that Wiersinga et al. reviewed the five crossover trials and identified that, despite adequate blinding, $48 \%$ of all hypothyroid patients preferred the combination of LT3 and LT4, 27\% preferred LT4 monotherapy, and 25\% had no preference [35]. Additionally, studies in which low or low normal TSH levels were often achieved reported benefits. Although this has been taken to suggest an overdose, the lower TSH levels may be related to the non-physiological nature of the LT3 replacement [3,26].

In general, it should not be assumed that combination therapy has no advantages over monotherapy until a well-designed long-term trial of combined physiological therapy with a sustained-release preparation or a dose of T3 three times a day is performed [3]. On the other hand, currently, all clinical practice guidelines agree that LT4 monotherapy should remain the treatment of choice in hypothyroid patients [35].

\section{Candidates for combined levothyroxine + liothyronine therapy}

It is necessary to ask whether there is any consensus from international associations regarding these emerging controversies about LT4 + LT3 combination therapy.

The European Thyroid Association (ETA) guidelines in 2012 [19] and the American Thyroid Association (ATA) guidelines in 2014 [15] both suggested that combination therapy with LT4 and LT3 could be considered as an experimental approach in hypothyroid patients treated with LT4, who have persistent complaints despite having serum TSH values within the reference range, provided coexisting autoimmune diseases have been ruled out [35].

It is important to note that for women of childbearing age using combined LT4 and LT3 therapy, foetal neurogenesis is primarily dependent on maternal FT4 levels at up to 16-18 weeks of gestation; therefore, regimens containing an excessive amount of LT3 can cause hypothyroxinaemia, which would then be associated with impaired foetal neurodevelopment [36]. There is insufficient data on foetal consequences of LT4 + LT3 combination therapy in pregnant women. Consequently, it seems prudent for safety reasons to refrain from combination therapy in pregnant women [19].

There is still concern about the long-term safety of LT4 + LT3 combination therapy, including cardiovascular safety, because ETA guidelines in 2012 established that the increase of serum-free T3 may provoke cardiac arrhythmias in susceptible patients [19].

However, the results of a 17-year population-based observational study based on the use of LT3 in Scotland have been reassuring. Compared with patients taking LT4 alone $(\mathrm{n}=33,955)$, those using LT3 (with or without LT4, $\mathrm{n}=400$ ) had no increased risk of cardiovascular disease, atrial fibrillation, or fractures after adjusting for age [35]. In a long-term cohort study conducted in Sweden, the use of LT3 did not lead to an increase in the incidence of breast cancer, the incidence of any cancer, and all-cause mortality compared to the use of LT4. Surprisingly, there was evidence of lower mortality in LT3 users after dose adjustment, which was potentially attributed to an artifact of the underlying associations between dose and patient diagnosis [37].

Recent Italian guidelines support the ETA and ATA guidelines. Furthermore, they were able to establish that combined therapy may be indicated in thyroidectomized adult patients with persistent symptoms of hypothyroidism and postoperative serum T3 levels and a FT4/FT3 ratio lower than their preoperative values during LT4 monotherapy. The LT4 + LT3 dose during combination therapy should be between 13:1 and 20:1 by weight. To avoid possible adverse effects, the starting dose should be approximately 17:1 [38].

If a trial of combination therapy is being considered in an individual patient, it is important to set clear expectations [39]. These include explaining that this is a non-standard treatment for which the risks remain unclear, with the potential risk of accelerated osteoporosis, atrial fibrillation, and stroke. Likewise, it is important to emphasize the importance of adherence with medication and follow-up [17].

Furthermore, it should be clear that this is a potentially non-standard therapy with significant monetary cost, and treatment will be discontinued if no benefit is 
experienced within a reasonable period, i.e. 3-6 months [19].

On the other hand, the NICE (The new National Institute for Clinical Excellence) guideline does not recommend routinely offering LT3 for patients with hypothyroidism, alone or in combination with LT4, because there is insufficient evidence proving its benefits over LT4 monotherapy, and long-term adverse effects are uncertain. The guideline does not give a clear recommendation on the use of LT3, but it thus suggests that it may play a role in patients with symptoms of hypothyroidism despite adequate replacement with LT4, and that further investigation is required [26, 40].

Genetic variations in deiodinases are associated with altered thyroid function and adverse health outcomes [41]. Individuals with genetic variants in D2 [42] and the thyroid hormone transporter protein MCT10 [43] have shown a preference for combined LT4 + LT3 therapy with an additive effect. However, these studies had limitations due to multiple tests and small sample size. The discovery of the polymorphism of the D2 gene (Thr92Ala), including its association with subtle changes in thyroid hormone homeostasis, quality of life index, and response to combined LT4 + LT3 therapy, has sparked enthusiasm as a possible explanation of patient dissatisfaction [3]. By contrast, Appelhof et al. determined genotypes for D2-orfA-Gly3Asp and D2-Thr92Ala polymorphisms in 141 patients with primary hypothyroidism, adequately treated with levothyroxine monotherapy, and found that these polymorphisms do not explain differences in well-being, neurocognitive functioning, or appreciation of T4/T3 combination therapy in patients treated for hypothyroidism.

In this way, alternative strategies, such as a single-nucleotide polymorphism panel or the use of thyroid hormone metabolites or metabolomics, may provide a better understanding of thyroid status in tissues $[3,8]$.

A consensus (ETA-2021) has recently been established on evidence-based recommendations on the use of the LT4 + LT3 combination in the treatment of hypothyroidism. This consensus describes the criteria that patients must meet in order to be recruited and tested for combination therapy. After excluding other causes of persistent symptoms, patients who do not report relief of their symptoms with LT4 therapy should be recruited. Also, patients must be treated with at least $1.2 \mu \mathrm{g} / \mathrm{kg} /$ day of LT4 to be eligible, and patients who have low baseline levels of total serum T3 while taking LT4 monotherapy should be included in the tests, and results should be stratified according to the change in total $\mathrm{T} 3$ levels achieved with the combination therapy. On the other hand, they recommend the use of validated thyroid-related quality of life questionnaires (PRO and ThyPRO-39), which should be used in assessing the baseline level of dissatisfaction, and they suggest that future trials of combination therapy should consider including the genotyping of polymorphisms and must have adequate power to study the effect of this polymorphism on the results of the study [9].

The authors of the present narrative review propose a treatment algorithm, profiling the hypothyroid patient who could receive LT3 (Fig. 1).

\section{Dose, monitoring, and preparations to be used in combined therapy with levothyroxine and liothyronine}

A wide range of LT3 preparations are available, with tablet concentrations ranging from 5 to $50 \mu \mathrm{g}$. ETA guidelines recommend an initial ratio of 13:1-20:1 (LT4:LT3), which represents a dose of 5 or $10 \mu \mathrm{g}$ of LT3 for patients taking 100-200 $\mu \mathrm{g}$ of LT4. Cutting LT3 pills with a pill cutter would allow twice-a-day dosing, such as $2.5 \mathrm{ug}$ twice a day. Initially, the LT4 dose is usually reduced by 12.5 or $25 \mathrm{ug}$ in order to accommodate the addition of LT3. The therapeutic substitution of LT3 for LT4 has previously been calculated to approximately $1: 3$ ratios $[38,45]$.

A simple method (Tab. 1) to arrive at the desired dose relationship between LT4 and LT3 is as follows: Dose " $x$ " is the daily dose of LT4 in $\mu \mathrm{g}$ that results in a normal serum TSH value. The required daily dose of LT3 in $\mu \mathrm{g}$ (called " $\mathrm{y}$ ") is given by $\mathrm{y}=\mathrm{x}: 20$. The required daily dose of LT4 in $\mu \mathrm{g}$ (called " $\mathrm{z}$ ") is given by $\mathrm{z}=\mathrm{x}-3 \mathrm{y}$. Considering that LT4 can be administered once a day, the daily dose of LT3 should be divided, if possible, into two doses, i.e. one before breakfast and the larger one before sleeping. The rationale for dividing the daily dose of LT3 is the relatively short half-life of LT3, peak serum T3 values $2-4 \mathrm{~h}$ after ingestion, and a physiological diurnal variation in serum $\mathrm{T} 3$ with the zenith around 4 a.m. and nadir between 3 and 5 p.m. [35].

The ETA guideline (2012) recommends using LT4 tablets separate from LT3 tablets in combination therapy, because the combination tablets contain a LT4:LT3 dose ratio of 4:1,5:1, and 10:1; these ratios are significantly different from the recommended ratios of 13:1-20:1. If dose adjustments are necessary, it is also more convenient to change the dose of only one of the components, preferably LT3 [35].

Regarding the duration of treatment with the combination therapy, there exists very little evidence; the RCTs ranged from 5 to 52 weeks [45]. Currently consensus recommends that it would be reasonable to run a trial for at least 1 year, with interim outcome assessments at 3 and 6 months [8]. The fixed-term 


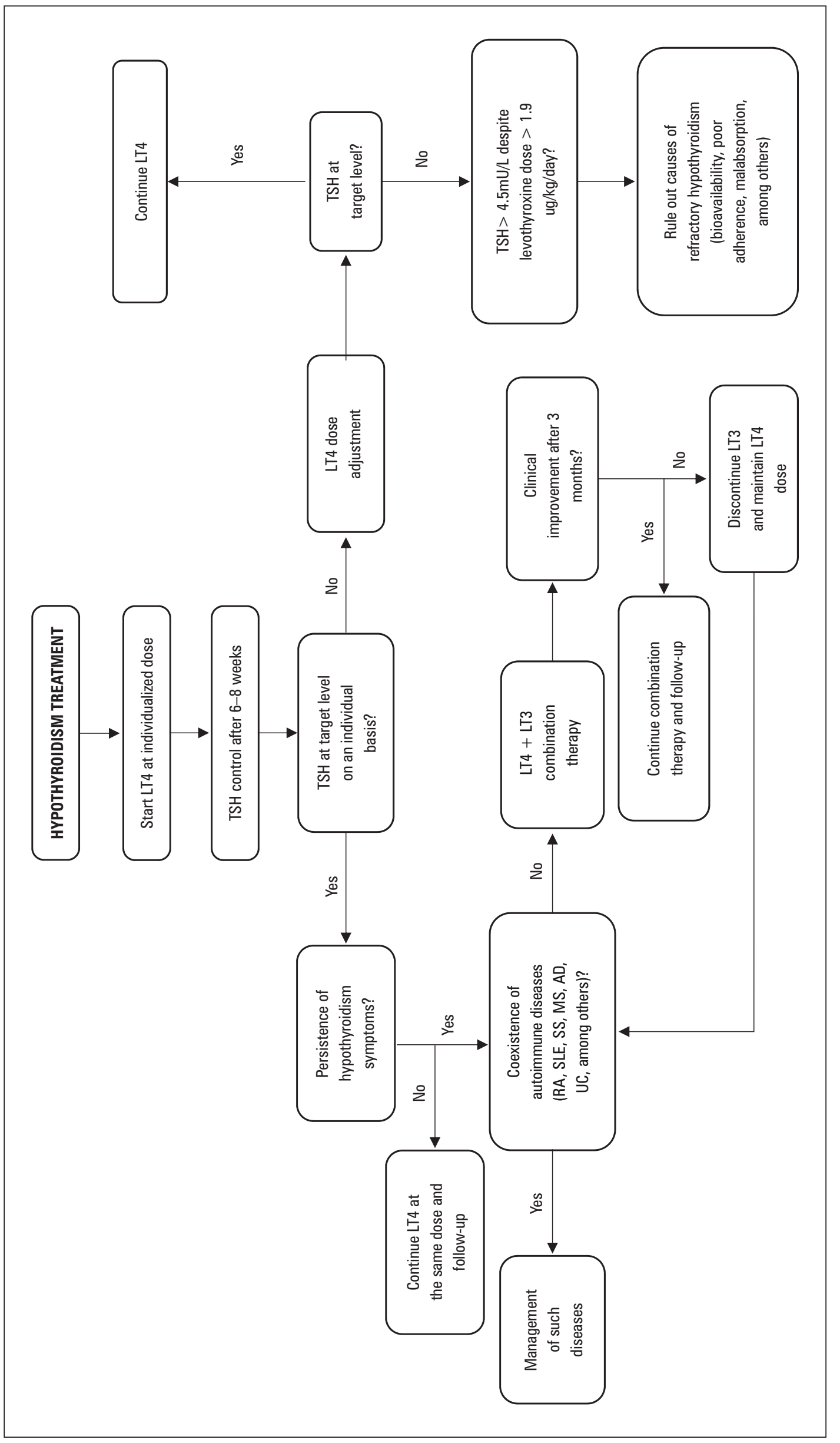

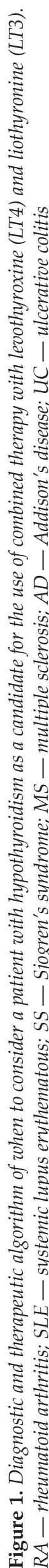


nature of the trial should be agreed with the patient before commencing, and there should be agreement that LT4 monotherapy will once again be the mainstay treatment if no significant benefit is seen [46].

Monitoring is challenging due to variations in serum T3 levels with current preparations. If the serum T3 peak (2-4 hours post-dose) is within reference values and TSH is not suppressed, it is probably a safe dose, although not all patients are satisfied in this range [5]. The goal of the LT4:LT3 combination should be to achieve a physiological T4/T3 ratio [35]. For patients in whom there is a long-term plan to continue treatment with LT3, monitoring of the regularity of the pulse and the conduct of an electrocardiogram to rule out atrial fibrillation are recommended at each medical visit, together with a bone densitometry in postmenopausal women every 3 years [9].

Response is commonly assessed through the patient's self-reporting of improvement, although questionnaires are used by some clinicians, e.g. ThyPRO. It is important to assess the response to be able to stop treatment if it is not effective or if the effect has been lost over time [9].

\section{Conclusion}

Combination therapy with LT3 and LT4 should be individualized for patients with primary hypothyroidism who receive LT4, those who have a target TSH concentration, and those with persisting symptoms, assessing its risks/benefits. Likewise, more studies are needed to be able to identify subgroups of hypothyroid patients who may benefit from the use of LT3, in addition to treatment with LT4, probably through the identification of new biomarkers or genetic polymorphisms.

\section{References}

1. Unnikrishnan AG, Kalra S, Sahay RK, et al. Prevalence of hypothyroidism in adults: An epidemiological study in eight cities of India. Indian J Endocrinol Metab. 2013; 17(4): 647-652, doi: 10.4103/2230-8210.113755, indexed in Pubmed: 23961480.

2. Santago C. Prevalence of thyroid dysfunction in the elderly population of an outpatient clinic. Acta Méd Colomb. 2018; 43(1): 24-30.

3. Taylor PN, Eligar V, Muller I, et al. Combination Thyroid Hormone Replacement; Knowns and Unknowns. Front Endocrinol (Lausanne). 2019; 10: 706, doi: 10.3389/fendo.2019.00706, indexed in Pubmed: 31695677.

4. Heald A, Livingston M, Hughes D. Management of Patients Symptomatically Unresponsive to Levothyroxine: Natural Desiccated Thyroid Extract or the Combination of Levothyroxine and Liothyronine? A Research Priority. Exp Clin Endocrinol Diabetes. 2020; 128(9): 596-598, doi: 10.1055/a-1062-6167, indexed in Pubmed: 31820425.

5. Mitchell AL, Hegedüs L, Žarković M, et al. Patient satisfaction and quality of life in hypothyroidism: An online survey by the british thyroid foundation. Clin Endocrinol (Oxf). 2021; 94(3): 513-520, doi: 10.1111/cen.14340, indexed in Pubmed: 32978985.

6. Peterson SJ, Cappola AR, Castro MR, et al. An Online Survey of Hypothyroid Patients Demonstrates Prominent Dissatisfaction. Thyroid. 2018; 28(6): 707-721, doi: 10.1089/thy.2017.0681, indexed in Pubmed: 29620972.

7. Wartofsky L. Combination L-T3 and L-T4 therapy for hypothyroidism. Curr Opin Endocrinol Diabetes Obes. 2013; 20(5): 460-466, doi: 10.1097/01.med.0000432611.03732.49, indexed in Pubmed: 23974776.
8. Celi FS, Zemskova M, Linderman JD, et al. Metabolic effects of liothyronine therapy in hypothyroidism: a randomized, double-blind crossover trial of liothyronine versus levothyroxine. J Clin Endocrinol Metab. 2011; 96(11): 3466-3474, doi: 10.1210/jc.2011-1329, indexed in Pubmed: 21865366.

9. Jonklaas J, Bianco AC, Cappola AR, et al. Evidence-Based Use of Levothyroxine/Liothyronine Combinations in Treating Hypothyroidism: A Consensus Document. Thyroid. 2021; 31(2): 156-182, doi: 10.1089/thy.2020.0720, indexed in Pubmed: 33276704.

10. Taylor PN, Razvi S, Muller I, et al. Liothyronine cost and prescriptions in England. Lancet Diabetes Endocrinol. 2019; 7(1): 11-12, doi: 10.1016/S2213-8587(18)30334-6, indexed in Pubmed: 30577888.

11. Fliers E, Kalsbeek A, Boelen A. Beyond the fixed setpoint of the hypothalamus-pituitary-thyroid axis. Eur J Endocrinol. 2014; 171(5): R197-R208, doi: 10.1530/EJE-14-0285, indexed in Pubmed: 25005935.

12. Feldt-Rasmussen U, Effraimidis G, Klose M. The hypothalamus-pituitary-thyroid (HPT)-axis and its role in physiology and pathophysiology of other hypothalamus-pituitary functions. Mol Cell Endocrinol. 2021; 525: 111173, doi: 10.1016/j.mce.2021.111173, indexed in Pubmed: 33549603.

13. Ortiga-Carvalho TM, Chiamolera MI, Pazos-Moura CC, et al. Hypothalamus-Pituitary-Thyroid Axis. Compr Physiol. 2016; 6(3): 1387-1428, doi: 10.1002/cphy.c150027, indexed in Pubmed: 27347897.

14. Larsen PR, Zavacki AM. The role of the iodothyronine deiodinases in the physiology and pathophysiology of thyroid hormone action. Eur Thyroid J. 2012; 1(4): 232-242, doi: 10.1159/000343922, indexed in Pubmed: 23750337

15. Jonklaas J, Bianco AC, Bauer AJ, et al. American Thyroid Association Task Force on Thyroid Hormone Replacement. Guidelines for the treatment of hypothyroidism: prepared by the american thyroid association task force on thyroid hormone replacement. Thyroid. 2014; 24(12): 1670-1751, doi: 10.1089/thy.2014.0028, indexed in Pubmed: 25266247.

16. Peeters RP, van Toor H, Klootwijk W, et al. Polymorphisms in thyroid hormone pathway genes are associated with plasma TSH and iodothyronine levels in healthy subjects. J Clin Endocrinol Metab. 2003; 88(6): 2880-2888, doi: 10.1210/jc.2002-021592, indexed in Pubmed: 12788902.

17. Luongo C, Dentice M, Salvatore D. Deiodinases and their intricate role in thyroid hormone homeostasis. Nat Rev Endocrinol. 2019; 15(8): 479-488, doi: 10.1038/s41574-019-0218-2, indexed in Pubmed: 31160732.

18. Bianco AC, Kim BS. Pathophysiological relevance of deiodinase polymorphism. Curr Opin Endocrinol Diabetes Obes. 2018; 25(5): 341-346, doi: 10.1097/MED.000000000000428, indexed in Pubmed: 30063552.

19. Wiersinga WM, Duntas L, Fadeyev V, et al. 2012 ETA Guidelines: The Use of L-T4 + L-T3 in the Treatment of Hypothyroidism. Eur Thyroid J. 2012; 1(2): 55-71, doi: 10.1159/000339444, indexed in Pubmed: 24782999.

20. Goldman JM, Line BR, Aamodt RL, et al. Influence of triiodothyronine withdrawal time on $131 \mathrm{I}$ uptake postthyroidectomy for thyroid cancer J Clin Endocrinol Metab. 1980; 50(4): 734-739, doi: 10.1210/jcem-50-4-734, indexed in Pubmed: 7364930.

21. Escobar-Morreale HF, del Rey FE, Obregón MJ, et al. Only the combined treatment with thyroxine and triiodothyronine ensures euthyroidism in all tissues of the thyroidectomized rat. Endocrinology. 1996; 137(6): 2490-2502, doi: 10.1210/endo.137.6.8641203, indexed in Pubmed: 8641203

22. Peterson SJ, McAninch EA, Bianco AC. Is a Normal TSH Synonymous With "Euthyroidism" in Levothyroxine Monotherapy? J Clin Endocrinol Metab. 2016; 101(12): 4964-4973, doi: 10.1210/jc.2016-2660, indexed in Pubmed: 27700539.

23. Jonklaas J, Davidson B, Bhagat S, et al. Triiodothyronine levels in athyreotic individuals during levothyroxine therapy. JAMA. 2008; 299(7): 769-777, doi: 10.1001/jama 299.7769, indexed in Pubmed: 18285588.

24. Gereben B, McAninch EA, Ribeiro MO, et al. Scope and limitations of iodothyronine deiodinases in hypothyroidism. Nat Rev Endocrinol. 2015; 11(11): 642-652, doi: 10.1038/nrendo.2015.155, indexed in Pubmed: 26416219.

25. Werneck de Castro JP, Fonseca TL, Ueta CB, et al. Differences in hypothalamic type 2 deiodinase ubiquitination explain localized sensitivity to thyroxine. J Clin Invest. 2015; 125(2): 769-781, doi: 10.1172/JCI77588, indexed in Pubmed: 25555216.

26. Leese GP. Nice guideline on thyroid disease: where does it take us with liothyronine? Thyroid Res. 2020; 13: 7, doi: 10.1186/s13044-020-00081-y, indexed in Pubmed: 32489426.

27. Okosieme O, Gilbert J, Abraham P, et al. Management of primary hypothyroidism: statement by the British Thyroid Association Executive Committee. Clin Endocrinol (Oxf). 2016; 84(6): 799-808, doi: 10.1111/cen.12824, indexed in Pubmed: 26010808.

28. Escobar-Morreale HF, Obregón MJ, Escobar del Rey F, et al. Replacement therapy for hypothyroidism with thyroxine alone does not ensure euthyroidism in all tissues, as studied in thyroidectomized rats. J Clin Invest. 1995; 96(6): 2828-2838, doi: 10.1172/JCI118353, indexed in Pubmed: 8675653. 
29. Jonklaas J, Davidson B, Bhagat $S$, et al. Triiodothyronine levels in athyreotic individuals during levothyroxine therapy. JAMA. 2008; 299(7): 769-777, doi: 10.1001/jama.299.7.769, indexed in Pubmed: 18285588.

30. Gullo D, Latina A, Frasca F, et al. Levothyroxine monotherapy cannot guarantee euthyroidism in all athyreotic patients. PLoS One. 2011; 6(8): e22552, doi: 10.1371/journal.pone.0022552, indexed in Pubmed: 21829633.

31. Ito M, Miyauchi A, Morita S, et al. TSH-suppressive doses of levothyroxine are required to achieve preoperative native serum triiodothyronine levels in patients who have undergone total thyroidectomy. Eur J Endocrinol. 2012; 167(3): 373-378, doi: 10.1530/EJE-11-1029, indexed in Pubmed: 22711760.

32. McAninch EA, Rajan KB, Miller CH, et al. Systemic Thyroid Hormone Status During Levothyroxine Therapy In Hypothyroidism: A Systematic Review and Meta-Analysis. J Clin Endocrinol Metab. 2018 [Epub ahead of print], doi: 10.1210/jc.2018-01361, indexed in Pubmed: 30124904.

33. Lee YKi, Lee H, Han S, et al. Association between Thyroid-Stimulating Hormone Level after Total Thyroidectomy and Hypercholesterolemia in Female Patients with Differentiated Thyroid Cancer: A Retrospective Study. J Clin Med. 2019; 8(8), doi: 10.3390/jcm8081106, indexed in Pubmed: 31349714.

34. Ito M, Miyauchi A, Hisakado M, et al. Biochemical Markers Reflecting Thyroid Function in Athyreotic Patients on Levothyroxine Monotherapy. Thyroid. 2017; 27(4): 484-490, doi: 10.1089/thy.2016.0426, indexed in Pubmed: 28056660.

35. Wiersinga WM. T4 + T3 combination therapy: any progress? Endocrine. 2019; 66(1): 70-78, doi: 10.1007/s12020-019-02052-2, indexed in Pubmed: 31617166.

36. Foeller ME, Silver RM. Combination Levothyroxine + Liothyronine Treatment in Pregnancy. Obstet Gynecol Surv. 2015; 70(9): 584-586, doi: 10.1097/OGX.0000000000000217, indexed in Pubmed: 26403562.

37. Planck $\mathrm{T}$, Hedberg $\mathrm{F}$, Calissendorff $\mathrm{J}$, et al. Liothyronine Use in Hypothyroidism and its Effects on Cancer and Mortality. Thyroid. 2021; 31(5): 732-739, doi: 10.1089/thy.2020.0388, indexed in Pubmed: 33040688.

38. Biondi B, Bartalena L, Chiovato L, et al. Recommendations for treatment of hypothyroidism with levothyroxine and levotriiodothyronine: a 2016 position statement of the Italian Society of Endocrinology and the Ital- ian Thyroid Association. J Endocrinol Invest. 2016; 39(12): 1465-1474, doi: 10.1007/s40618-016-0511-z, indexed in Pubmed: 27473077.

39. Zavaleta MJ, Arroyo JC, Gutiérrez FE, et al. T3 therapy in hypothyroidism. Still more questions than answers. Arch Endocrinol Metab. 2021; 65(3): 392-393, doi: 10.20945/2359-3997000000378, indexed in Pubmed: 34191413.

40. Vasileiou M, Gilbert I, Fishburn S, et al. Guideline Committee. Thyroid disease assessment and management: summary of NICE guidance. BMJ. 2020; 368: m41, doi: 10.1136/bmj.m41, indexed in Pubmed: 31996347.

41. Taylor PN, Peeters R, Dayan CM. Genetic abnormalities in thyroid hormone deiodinases. Curr Opin Endocrinol Diabetes Obes. 2015; 22(5): 402-406, doi: 10.1097/MED.0000000000000180, indexed in Pubmed: 26192703.

42. Panicker V, Saravanan P, Vaidya B, et al. Common variation in the DIO2 gene predicts baseline psychological well-being and response to combination thyroxine plus triiodothyronine therapy in hypothyroid patients. J Clin Endocrinol Metab. 2009; 94(5): 1623-1629, doi: 10.1210/jc.2008-1301, indexed in Pubmed: 19190113.

43. Carlé A, Faber J, Steffensen R, et al. Hypothyroid Patients Encoding Combined MCT10 and DIO2 Gene Polymorphisms May Prefer L-T3 + L-T4 Combination Treatment - Data Using a Blind, Randomized, Clinical Study. Eur Thyroid J. 2017; 6(3): 143-151, doi: 10.1159/000469709, indexed in Pubmed: 28785541.

44. Appelhof BC, Peeters RP, Wiersinga WM, et al. Polymorphisms in type 2 deiodinase are not associated with well-being, neurocognitive functioning, and preference for combined thyroxine/3,5,3'-triiodothyronine therapy. J Clin Endocrinol Metab. 2005; 90(11): 6296-6299, doi: 10.1210/jc.2005-0451, indexed in Pubmed: 16144953.

45. Madan R, Celi FS. Combination Therapy for Hypothyroidism: Rationale Therapeutic Goals, and Design. Front Endocrinol (Lausanne). 2020 11: 371, doi: 10.3389/fendo.2020.00371, indexed in Pubmed: 32733377.

46. Saravanan P, Simmons DJ, Greenwood R, et al. Partial substitution of thyroxine (T4) with tri-iodothyronine in patients on T4 replacement therapy: results of a large community-based randomized controlled trial. J Clin Endocrinol Metab. 2005; 90(2): 805-812, doi: 10.1210/jc.2004-1672, indexed in Pubmed: 15585551. 